\title{
Sealability of Filled and Unfilled Fissure Sealants using Two Different drying agents

\author{
*Essam Bakry
}

\begin{abstract}
To evaluate and compare the effect of two different drying agents (PrimaDry and Ethyl alcohol 95\% concentration.) on microleakage of filled (Ultraseal XT plus) and unfilled (Helioseal) fissure sealants in vivo and in vitro. A total of 60 extracted human upper and lower first premolars from 15 adult healthy patients were divided into 2 groups 30 in vivo (Teeth were extracted 3 months after sealant application), and 30 in vitro (Teeth were stored 3 months in normal saline) each group were subdivided into 2 subgroups 15 sealed with filled (Ultraseal XT plus), while the other 15 were sealed with unfilled (Helioseal), each subgroup was subdivided into 3 classes composed of (5) premolars according to drying agent used: B0) without drying agent. B1) PrimaDry B2) Ethyl alcohol 95\% concentration. After extraction teeth were thoroughly washed with water, cleaned with non-fluoridated (FL) prophylaxis paste. The unsealed were sealed after 3 months of storage in saline solution (in vitro). The occlusal surfaces were opened using tapered diamond fissure burs confined to enamel. Fissure sealant application was done following the manufacturer's instructions. The drying agent was applied after etching and before sealant application. Dye penetration method with methylene blue dye was used for microleakage assessment. The specimens were immersed in $1 \%$ methylene blue for $24 \mathrm{~h}$ in order to allow dye penetration into possible gaps in the tooth-sealant interface. The teeth were sectioned longitudinally in using diamond saw, each section cleaned, examined and photographed under stereomicroscope at $\times 20$ magnifications to assess dye penetration at the margins of the restorations. Two-way ANOVA was used for statistical analysis. In vivo results revealed that unfilled (Helioseal) achieved significant less microleakage than filled (Ultraseal XT plus) without any drying agent and PrimaDry with either filled or unfilled achieved the least microleakage. While in vitro results showed that both PrimaDry with Filled (Ultraseal XT plus) and Ethyl alcohol $95 \%$ concentration with Unfilled (Helioseal) achieved the least microleakage without significant statistical difference. Use of Ultraseal XT plus system according to manufacturer's directions showed the least microleakage both in vivo and in vitro. Use of PrimaDry increased the efficiency of both filled and unfilled fissure sealant.
\end{abstract}

\section{Introduction}

The effectiveness of sealants hinges on their ability to isolate pits \& fissures from the combination of bacteria, their nutrients, and acidic metabolic products. Microleakage has been defined as clinically undetectable passage of bacteria, fluids, molecules, or ions between the cavity walls and the completeness of the resin infiltration process affects microleakage and is diffusion dependent. (Hatibovic - Kofman et al., 2001).

Fissure sealants were initially developed to seal sound pits and fissures, creating a mechanical barrier between the enamel and the oral environment and therefore preventing demineralization and the development of caries lesions. Fissure sealants are now increasingly being used therapeutically as well as preventively, where non cavitated and cavitated caries lesions are being sealed in order to arrest the caries process. (Celiberti et al., 2010).

\begin{tabular}{llll}
\hline *Fasciomaxillary & Department & Ahmed & Maher \\
Teaching Hospital & & &
\end{tabular}

\section{Review:}

Fissure sealant and microleakage Hotibovic-Kofman et al., (1998), studied the microleakage of sealants after conventional, bur, and air abrasion preparation of pits and fissures. They divided Seventy-two extracted molars randomly into three groups. In group I, 24 teeth were prepared by pumicing and acid etching with $37 \%$ phosphoric acid. In group II, 24 teeth were prepared with a round bur in a low-speed handpiece and then acid etched. In group III, 24 teeth were prepared by high-speed microabrasion using $50 \mu \alpha$ alumina particles in a $\mathrm{KCP}$ 2000 machine. In each group, 12 teeth were sealed with a filled sealant and 12 teeth with an unfilled sealant. All the teeth were stored in artificial saliva for 7 days and thermocycled for 2000 cycles. Teeth were then sealed apically and coated with nail varnish $1 \mathrm{~mm}$ from the margins and stained in $1 \%$ methylene blue for 24 hours. Each tooth was sectioned in three locations and ranked (03 ) for microleakage. They found that $66 \%$ of 216 sections showed no leakage, and $26 \%$ showed minimal leakage. The major finding of this study is that bur preparation followed by acid etching produced sealants with less 


\section{Essam Bakry}

microleakage than conventional or air abrasion tooth preparation also they found that unfilled sealant had less microleakage than filled sealant as the latter is more viscous so poorer adaptation to enamel and incomplete penetration to the bottoms of the pits and fissures resulting in decreased retention. They concluded that superior results were obtained when the tooth surfaces were prepared by a bur and the unfilled was superior to the filled sealants.

Kwon and Park (2006), Studied SEM and microleakage evaluation of 3 flowable composites as sealants without using bonding agents. They used three flowable composites (Filtek Flow, Tetric Flow, Charmfil Flow) and a filled sealant (Ultraseal XT Plus). The patterns of resin tag formation in the 4 sealant materials were compared using scanning electron microscopy. They found that the three flowable composites and the filled sealant showed a similar resin tag formation pattern and the 3 flowable composites showed significantly more microleakage in each group than the filled sealant. The level of microleakage was similar in the 3 flowable composites. They concluded that the use of the filled sealant is more effective in sealing mechanically prepared occlusal fissures in comparison to the flowable composites.

\section{Enamel preparation}

Rix et al., (1994), evaluated pit and fissure sealant application using a drying agent through a clinical investigation. They assessed the retention of pit and fissure sealants with and without the use of a post-etching drying agent in pediatric dental patients. Patients with four fully erupted; non carious first permanent molars participated. Patients 5-12 years of age each received four sealants, two with and two without a drying agent. Sealants were placed by three dental operators. Assignment for sealant application (with and without drying agent) was based on a computer generated randomization table. Sealant application involved cleansing of teeth with a non-fluoridated prophylaxis paste followed by rinsing 60 seconds with air/water spray, enamel conditioning with $40 \%$ phosphoric acid for 60 seconds, application of drying agent for 5 seconds, application of sealant material, and light curing for 60 seconds. Modified USPHS (Ryge) criteria were used to evaluate interfacial staining, secondary caries, marginal integrity, and surface texture. Sealant retention was evaluated as fully retained or lost (failed). All patients returned for the 12-month recall visit. Four evaluators reported a $91 \%$ overall retention rate. Of the teeth sealed without a drying agent, there was a $13 \%$ failure rate compared to $5 \%$ failure rate for the teeth sealed with a drying agent.

Adams et al, (2000), studied the effect of drying agent use on sealant penetration. Sealants are an effective method to prevent pit and fissure caries, however, retention and efficacy are influenced by the ability of resin to wet etched enamel and penetrate narrow fissures. This study evaluated the effect of a drying agent (primaDry) on penetration of a light cure sealant Ultraseal XT plus. They used about twenty-four extracted mandibular molars and applied acid- etching (15 sec.), rinsing (25 sec.), and air shield $(15 \mathrm{sec}$.) prior to sealant application then light curing. They concluded that drying agent enhances the sealant penetration into pits and fissures. Geiger et al., (2000), studied the mechanical preparation and filling level of fissure sealant. The objective of this study was to assess the effect of different modes of fissure preparation and filling level on the quality of pits and fissure sealant. Various modes of fissure preparation in combination with two filling levels were examined. A total of 90 caries-free extracted human molar teeth were divided into three groups according to fissure preparation: (a) no mechanical preparation; (b) mechanical preparation with a round carbide bur; and (c) mechanical preparation with a tapered fissure diamond bur. All fissures were acid-etched and each group was subdivided according to filling level, either to the border or overfilled, using Helioseal (Vivadent, Schaan, and Lichtenstein) as a sealant material. Microleakage was assessed, combining occlusal loading and thermocycling, prior to staining with $0.5 \%$ Basic Fuchsin. After sectioning, teeth were examined and photographed in a reflected light microscope. Microleakage and sealant penetration depths were measured for each section. They found that fissure sealant penetration and retention were significantly improved by mechanical preparation, preferably with a tapered diamond bur, and filling just to the border. Overfilling of fissures should be avoided.

Hatibovic - Kofman et al., (2001), studied the microleakage of three sealants following; conventional, bur, and air- abrasion preparation of pits and fissures. They compared the microleakage of filled sealant; Ultraseal XT plus with a drying agent; PrimaDry, a filled sealant (Prisma shield) and an unfilled sealant (Delton) after preparation of teeth using conventional, bur, air abrasion then teeth were cleaned and divided into three groups. 180 extracted teeth were cleaned and divided randomly into three groups. Group I was prepared using acid etch only. Group II was prepared with $1 / 4$ round burs in a low speed headpiece followed by acid etching. Group III was prepared with high-speed micro-abrasion. The teeth in each group were randomly assigned to one of the three subgroups according to the sealant applied. Subgroup A was sealed with a filled sealant using a drying agent, subgroup B was sealed with a filled sealant only, and subgroup $\mathrm{C}$ was sealed with an unfilled sealant. Microleakage was assessed using $1 \%$ methylene blue dye for $48 \%$ at $37 \%$. Each tooth was sectioned at 4 locations buccolingually and were ranked (0-3) for microleakage. The results showed that Ultraseal XT plus, the filled sealant with drying agent, revealed significantly less microleakage i 4

than the other two sealants, also air abrasion with acid etch showed significantly less microleakage than either bur with acid etch or acid etch alone. They concluded that Ultraseal XT plus was the most effective sealant for preventing microleakage in this study.

Duangthip et al., (2003), studied the effects of fissure cleaning methods, drying agents, and fissure morphology on microleakage and penetration ability of sealants in 


\section{Sealability of Filled and Unfilled ....}

vitro. The purpose of this study was to determine the effect of different cleaning methods and the use of postetching drying agents, as well as the influence of fissure types on the microleakage and penetration ability of fissure sealants. They found that no significant difference was found in microleakage and the penetration ability between the use of different cleaning methods and sealant placement with or without drying agents. They concluded that neither air abrasion with acid etching nor the use of post-etching drying agents decreased microleakage and improved the penetration ability of sealants significantly compared to the conventional sealant application. Fissure type was significantly related to the penetration ability of sealants. (Fig1)

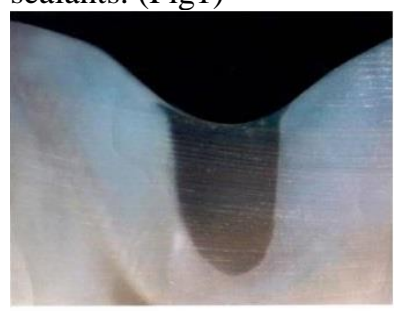

a) No microleakage

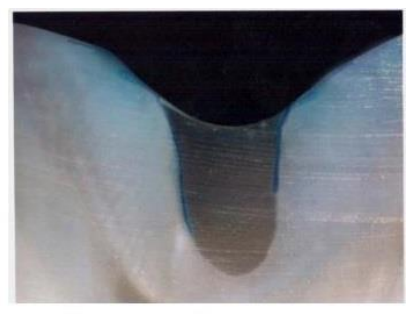

b) With microleakage
Fig.1:absence and presence of microloleakage under microscope.

\section{Material :}

1 Pit and fissure sealants: 1.1.1 Filled pit and fissure sealant. (Ultraseal XT plus) 1.1.2 Unfilled pit and fissure sealant. (Helioseal)

2 Drying agents: 1.2.1 PrimaDry. 1.2.2 Ethyl alcohol $95 \%$ conc.

\section{Methodology: \\ 1 Selection of participants In this study, fifteen young healthy patients were selected, their age range is (15-25) years, they were scheduled for extraction of upper and lower first premolars of both sides for orthognathic surgery. Fissure sealing was performed only for one upper premolar and one lower premolar on the same side, while the opposite upper and lower premolars will be sealed outside the patient's mouth after extraction. Patients were informed about the study, and agreed to give their consent to participate.}

2 Patient examination Past and present medical histories were obtained in the form of a printed systemic health questionnaire which was filled by all patients. Past and present dental histories were obtained including oral hygiene measures, periodontal status, frequency of teeth brushing and scaling. Clinical examination was done through intraoral inspection and careful exploration using blunt explorer. (Probe only restricted to remove plaque and/or food debris).

3 Grouping of teeth The teeth were divided into 2 groups in vivo and in vitro 30 teeth each. Each group was subdivided into 2 subgroups filled and unfilled of 15 teeth each. Each subgroup either in vivo or in vitro was subdivided into 3 equal divisions without drying agent (B0) (control group), primaDry (B1), and ethyl alcohol (B2).
4 Preparation \& collection of the specimens Both sealed and unsealed teeth were extracted 3 months following surgery, teeth were thoroughly washed with water, cleaned with non fluoridated (FL) prophylaxis paste. The unsealed were sealed outside the mouth (in vitro). Pit and fissure sealant application was done following the manufacturer's instructions. The drying agents were applied after etching and before sealant application. In our vivo study sealant application procedures done as followed; After teeth selection plaque and/or debris removed using pumice polishing paste with occlusal brush, occlusal fissures of teeth were opened using tapered fissure diamond burs depth confined to enamel, then after rinsing each tooth was isolated with cotton rolls, dried, etched either with (echo-etch) or (ultraetch) for 15 seconds, rinsed 5 seconds until complete removal of all etching material, then cotton rolls removed and replaced by dry ones, and air dried until frosty white appearance Each drying agent applied 5 seconds, then gently dried, Sealant either (Filled) or (unfilled) was applied with applicator supplied by manufacturer, immediate curing with light emitting diode (C5) blue phase (Ivoclar, Vivadent) after placement for 20 seconds, isolation removed and area rinsed well, occlusion checked with articulating paper necessary adjustments done. The same procedures were repeated to freshly extracted teeth (in vitro).

5 Specimen preparation: Dye penetration method using methylene blue dye was used in this study for microleakage assessment Teeth were coated from root apecies up till crowns with thin layer of pink modeling wax leaving $2 \mathrm{~mm}$ of enamel around sealant uncovered, then by thin layer of tin foil, then by 2 thin layers of nail varnish and the teeth were allowed to dry then specimens were immersed in $1 \%$ methylene blue for $24 \mathrm{~h}$ in order to allow dye penetration into possible gaps in the toothsealant interface. The coatings were then stripped off, the teeth rinsed thoroughly under running tap water, then sectioned longitudinally in buccolingual direction and three $1 \mathrm{~mm}$-thick sections (distal, central and mesial) were obtained from each tooth using diamond saw ionEdficaenta (Hi flex, USA) under running tap water. 6 Microleakage assessment: The dye penetration technique of microleakage assessment demonstrates a discrete, high contrast marking at the sealant-enamel interface. In this study, the linear measurement system for identifying the diffusion of dye penetration was used. The advantages of the linear measuring system are that more objective measurements are obtained. This quantitative data allows the use of a more powerful parametric statistical analysis. Specimens from each group for resin enamel interface evaluation were examined. Each section was, examined, and photographed in the stereomicroscope (Olympus stereomicroscope), then (Image J 1.41, NIH, USA) software was used for image analysis and linear measurement for dye penetration was carried out.i 6 


\section{Essam Bakry}

\section{Statistical analysis:-}

Data were presented as mean and standard deviation (SD) values. Regression model with Two-way Analysis of Variance (ANOVA) was used in testing significance for the effect of sealant, drying and their interaction on mean microleakage. Tukey's post-hoc test was used for pairwise comparison between the means when ANOVA test is significant. For detailed comparisons between the groups, one-way ANOVA was used to compare between the three drying conditions and Student's t-test was used to compare between the two sealants. The significance level was set at $\mathrm{P} \leq 0.05$. Statistical analysis was performed with SPSS $16.0^{\circledR}$ (Statistical Package for Scientific Studies) for Windows.

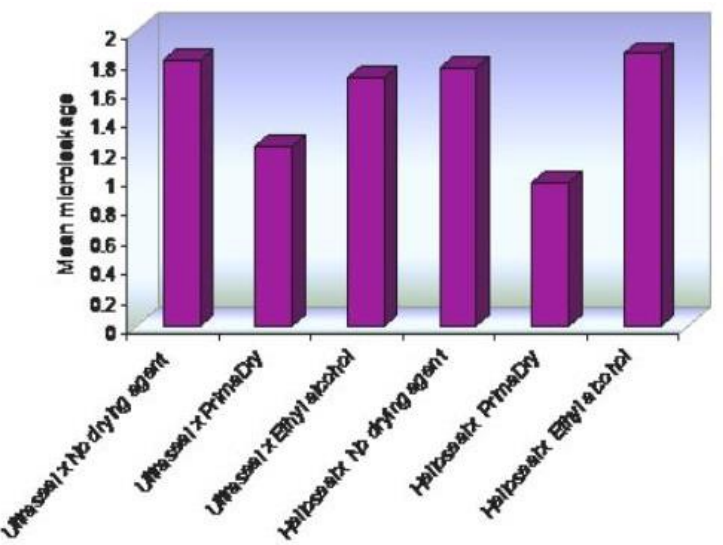

Fig:2 bar chart showingcomparison between the different interactions of sealants and drying agents

\section{Results}

In vivo results: the results showed that the statistically significantly highest mean microleakage was between Helioseal with Ethyl alcohol $(1.85 \mu \mathrm{m})$, followed by Ultraseal with no drying agent $(1.81 \mu \mathrm{m})$, then Helioseal with no drying agent $(1.75 \mu \mathrm{m})$ and then Ultraseal with Ethyl alcohol $(1.69 \mu \mathrm{m})$,with statistically unsignificant difference between them at $\mathrm{P}=0.008$. The statistically significantly lowest microleakage values were obtained with (Ultraseal with PrimaDry $(1.22 \mu \mathrm{m})$ and Helioseal with PrimaDry $(0.97 \mu \mathrm{m})$ with no statistically significant difference between the two groups ( $\mathrm{p}$-value $=0.008$ )

In vitro results :- The results showed that UltraSeal with no drying agent showed the statistically significantly highest mean microleakage $(1.34 \mu \mathrm{m})$. This was followed by UltraSeal with Ethyl alcohol $(0.98 \mu \mathrm{m})$. HelioSeal with no drying agent showed lower value $(0.80 \mu \mathrm{m})$. HelioSeal with PrimaDry $(0.55 \mu \mathrm{m})$. While the lowest values of mean microleakage were obtained by HelioSeal with Ethyl alcohol $(0.29 \mu \mathrm{m})$ and UltraSeal with PrimaDry $(0.28 \mu \mathrm{m})$ with unsignificant statistical difference. Results of stereomicroscopic evaluations of resin enamel bonded interface. Stereomiroscope evaluation for both groups of filled and unfilled sealants in vivo and in in vitro was carried out. Sections from each tooth in each group was examined, photographed and linear dye penetration was measured via software image analysis ${ }^{\circledR}$ SPSS, Inc., Chicago, IL, USA.

\section{Disscussion}

Pits and fissures sealants were introduced in 1967 and their effectiveness was recognized by the ADA in 1971. Despite their quick and easy setting, sealant success depends on meticulous operator technique. The parameters directly tested here included the method of fissure preparation (diamond bur) and preparation of enamel (etching or not). These steps in fissure sealing appeared to be of major importance for sealant retention and longevity, yet the optional clinical procedure was still not well established. Several in vitro studies have assessed the effectiveness of sealants after different kinds of enamel preparation. The evaluation of marginal leakage gave a good idea of the sealant capacity in preventing bacterial invasion and consequently reduces the risk of secondary caries development. Using a sharp probe or scraping the pits and fissures did not achieve the goal of sufficient debridement and complete penetrations of the acid etch and subsequently of the sealant rarely occurred with the conventional cleaning methods. Moreover, many studies reported a better efficiency of sealants when using the invasive technique. This technique provided certain advantages, such as better diagnosis of underlying decalcifications, higher retention rates and reduced risk of microleakage by widening and deepening the pits and fissures, eliminating organic material and plaque, and exposing more reactive tooth enamel, therefore enabling a thicker layer of sealant. Moreover, clinical evidence tended to support the merit of bur prepared surfaces, like in a 6-year controlled clinical study by Shapira and Eidelman 1986. (Lupi-Pegurier et al., 2003) Smoothing of the cavity walls and the absence of debris, which was more easily done with the diamond bur, could have played a central role in increasing sealant adhesion to enamel. (Geiger, et al., 2000) The clinically undetectable passage of bacteria, fl uids, molecules, or ions between the cavity wall and the applied restorative material, known as microleakage which is an important concern in restorative dentistry because of its clinical damages, such as secondary caries lesions, pulpal pathologies, postoperative pain and sensitivity and, consequently, the failure of the restorative procedure. In the case of pit and fissuresealants, the success of this technique can be hindered if the applied material cannot resist microleakage, resulting in the initiation and/or progression of caries under sealed surfaces, as well as increasing the difficulty of diagnosing and treating this lesion. In an attempt to improve the retention of sealants and decrease microleakage, mainly when the control of humidity is unsatisfactory, the association of adhesive systems and pits and fissures sealants was proposed. (Youssef, et al., 2006). In the present study tapered fissure diamond burs were used in preparation followed by phosphoric acid etching to increase the longevity of fissure sealants by improving sealant penetration and by decreasing marginal leakage, preventing bacterial invasion, and consequently reducing the risk of secondary caries development. (Geiger, et al., 2000)

Haws et al 1996 studied air abrasion and microleakage of pit and fissure sealants. The results of this study showed 


\section{Sealability of Filled and Unfilled ....}

that acid etching alone or in combination with air abrasion is a better option than air abrasion alone to prevent leakage of pit and fissure sealants. In order for the sealant to adhere the enamel surface needs to be etched, usually with an orthophosphoric acid liquid gel. Liquid and gel are equally effective in terms of surface penetration and sealant retention. Clinical studies indicate that a 15 seconds etch is adequate for sealant retention and no additional benefit received from longer etching times of 45 or 60 seconds (Locker et al 2003). Fissure enamel was considered resistant to etching. This etch resistance was not attributed to post-eruptive changes, but to a ring of aprismatic enamel aroundfi ssures' entrance and walls. Aprismatic enamel crystals exhibit an unidirectional orientation and are densely arranged. Etching this structure results in a relatively uniform dissolution and creation of limited porosity and resin penetration, manifested by short resinous tags. However, it was supposed that a greater tag number rather than that length is responsible for a stronger bond. An in vivo study demonstrated that the bond will succeed longer when a larger amount of high quality etching pattern is present (Paula and Adrian 2005). The dye penetration technique of microleakage assessment demonstrates a discrete, high contrast marking at the sealant enamel interface. In this study, the linear measurement system for identifying the diffusion of dye penetration was used. The advantages of the linear measuring system are that more objective measurements are obtained. This quantitative data allows the use of a more powerful parametric statistical analysis. (Jacob Lee 2003). In both in vivo and in vitro results, the UltraSeal XT with PrimaDry showed the best results of lowest values of mean microleakage followed by the Helioseal with primaDry which could be attributed to the significant effect of primaDry on microleakage shown in the present study and that was in agreement with Adams, et al., 2000 \& Hatibovic Kofman et al., 2001 who found that when PrimaDry was applied after acid etching and prior to sealant application. The drying agent is ethyl alcohol and it is thought to remove any residual moisture left after air drying the acid etched tooth surface. Pit and fissure sealants are generally hydrophobic, and acid etching improves the ability of the sealant to wet the enamel surface. The penetration of Ultraseal XT plus into fissures was reported to be significantly greater. By completely drying the acid-etched surface, Primadry may further improve the ability of the sealant to wet enamel allowing superior penetration and resin tag formation resulting in reduced microleakage. (Adams, et al., 2000) A consistent problem the clinician encounters is the moisture contamination of enamel surface. The Ultraseal XT sealant system solves this problem via the PrimaDry, which is a drying agent; an alcohol-based liquid with a hydrophilic monomer, the PrimaDry chases the water molecules in the pits \& fissures, volatizing them. This effect was evidenced by even frostier appearance of enamel after application. (Jacob Lee 2003). A clinical study of UltraSeal XT plus has been conducted in which the 2-year retention rate was $96.3 \%$. The application system of Ultraseal XT plus was easier to manage, the ease of this system may allow more rapid, controlled and accurate sealant application especially in the mouth (Hatibovic Kofman, et al., 2001). The results of in vivo study showed that sealant type had no statistically significant effect on mean microleakage; there was no statically significant difference between mean microleakages of the two sealants when used without drying agent. This result was in agreement with Xalabarde, et al., 1996 who found that there was no difference in penetration or adaptation of filled and unfilled sealants as without drying agent water in pits \& fissures may hinder the penetration of resin in micropores created by enamel etching, and in disagreement with the in vitro results which could be due to the numerous deficiencies of in vitro testing $\&$ the inherent problems in processing tooth samples. Regardless the drying agent used, UltraSeal showed statistically significantly higher mean microleakage than Helioseal, The results of this study are consistent with previous findings that showed statistically significant less microleakage with unfilled sealant. It is possible that the less viscous unfilled resin had greater penetration and adaptation resulting in less microleakage. Flanagan et al., 1988; Hatibovic Kofman et al., 1998; Stephan et al., 2000 found superior penetration into deep fissures with low viscosity sealants. Ruyter and Oysaed, 1987 believe that the unfilled resin has greater uptake of water and, consequently, undergoes greater hygroscopic expansion, minimizing the effect of the high coefficient of thermal expansion. However , Hansen and Asmussen. 1989 are skeptical that such hygroscopic expansion compensates completely and uniformly throughout the sealant, eliminating the detrimental effect of thermocycling. The advantages of unfilled sealant are its ability to wet the surface applied on, its rheological property to spread across the enamel entering its microspores (Gwinnett et al., 1967). This improved adaptation of adhesive at the interface manifests as greater resin tag penetration prior to light activated polymerization. As the filler content of the sealant increases its wetting ability decrease. (Jacob Lee 2003). Unfilled sealants showed superior penetration pattern especially following the acid etching. (Figuiredo and Pavarini 1996). The results of in vitro study showed that the Helioseal with ethyl alcohol showed the least microleakage as the Ultraseal with primaDry followed by Helioseal with primaDry which as not correlated with in vivo outcome. The results of in vitro microleakage studies are extremely variable, making conclusions difficult. (Hatibovic Kofman, et al., 2001), also most in vitro data do not strictly follow the same set of specifications or standards and this limits the comparability of results. There are not known strong correlations between laboratory testing and short-term or long-term clinical performance. (Shu-Fen Chuang et al., 2006)

\section{CONCLUSIONS}

Under the conditions of this study, the following conclusions could be drawn:1-Use of Ultraseal XT plus system according to manufacturer's directions showed the least microleakage both in vivo and in vitro.2-Use of 


\section{Essam Bakry}

PrimaDry increased the efficiency of both filled and unfilled fissure sealants.

RECOMMENDATIONS:

Use of PrimaDry may be recommended clinically before fissure sealant application. More investigations could be carried out with increasing the concentration of Ethyl alcohol to $(99 \%)$ or $100 \%$ (absolute ethyl alcohol) to reveal its ability to reach the efficiency of PrimaDry.

\section{REFERENCES}

1.Adams T, Frazier K, Browning WD. Effect of drying agent use on fissure sealant penetration. Journal of Dental Research 2000; 79: 189 (Abstr.363).

2. Boksman L, Carson B. Two year retention and caries rates of Ultraseal XT and Fluoroshield light - cured pit and fissure sealants. Journal of General Dentistry 1998; 46(Abstr.2): 184-7.

3. Choi JW, Drummond JL, Dooley R, Punwani I, Soh JM. The efficacies of primer on sealant shear bond strength. Journal of Dental Research 1996; 75: 180 (Abstr. 1299).

4. Feigal RJ, Musherure P, Gillespie B, LevyPolack M,Quelhas I, Hebling J. Improved sealant retention with bonding agents: a clinical study of two-bottle and single-bottle systems. Journal of Dental Research 2000; 79: 1850-1856.

5. Figeiredo MM, Pavarini A. Microscope evaluation of with and without inorganic particles and tag formations. Journal of Dental Research 1996; 75: 180 (Abstr.1289).

6. Geiger S.B, Gulayev S, Weiss E.I. Improving fissure sealant quality: mechanical preparation and filling level. journal of Dentistry 2000;28:407-412.

7. Glawer N. Chopra R. The effect of fissure morphology and eruption time on penetration and adaptation of pit and fissure sealants: An SEM study. Journal of Indian Social Pedodontic Preventive Dentistry 2008; 9:59-63.

8. Handelman SL, Buonocore MC, Schouter PC. Progress report of the effect of a fissure sealant on bacteria in dental caries. Journal of the American Dental Association 1973; 87:1198-1192.
9. Hassall D.C and Mellor A.C. The resin restoration: Indications, success and clinical technique. British Dental Journal 2001; 191: 358-362.

10.Hatibovic-Kofman S, Butter SA, Sadek H. Microleakage of three sealants following conventional, bur, and air abrasion preparation of pits and fissures. International Journal of Pediatric Dentistry 2001; 11(6): 409-416.

11.Hatibovic-Kofman S,Wright GZ, Braverman I. Microleakage of sealants after conventional, bur, and air abrasion preparation of pits and fissures. Journal of Pediatric Dentistry 1998; 20: 173-176.

12.Haws SM, Oliveira ML,Vargas MA, Kanellis MJ. Air abrasion and microleakage of pit and fissure sealants. Journal of Dental Reseach 1996; 75: 180 (Abstr. 1301)

13.Hevinga M A, Opdam NJM, Frencken JE, Bronkhorst EM, Truin GJ. Microleakage and sealant penetration in contaminated carious fissures. Journal of Denistry 2007; 35:909914.

14.Kuyiuu E, Styner D, Scherer W, Renwicek E. Evaluation of three adhesive resins for use as pit and fissure sealants. Journal of Dental Research 1996 ; 75: 180 (Abstr. 1302).

15.Kwon HB, Park KT. SEM and microleakage evaluation of three flowable composites as sealants without using bonding agents.Journal of Pediatric Dentistry2006;28(1):48-53.

16.Leverette DH, Handelman SI, Brenner CM, Iker HP. Use of sealants in the prevention and early treatment of carious lesions: cost analysis. Journal of the American Dental Association 1983; 106: 39-42.

17. Locker D, and Jokovic A. The use of pit and fissure sealants in preventing caries in permanent dentition of children. British Dental Journal 2003; 195:375-378.

18. Lupi-Pegurier L, Bertrand MF, Muller-Bolla M, Rocca JP,Bolla M. Comparative study of microleakage of a pit and fissure sealant placed after preparation by Er:YAG laser in permanent molars. Journal of Dentistry for Children 2003; 70:134-138. 


\section{Sealability of Filled and Unfilled ....}

19.Mary Govoni. Success with pit and fissure sealants. Dental Assistant; May/June 2002; 71, 3; ProQuest medical library pg.8

20.Masashi Miyazaki, Naoshi Hirohata, Kenji Takagaki, Hideo Onosea, Keith Moore B. The Influence of self etching primer drying time on enamel bond strength of resin composites. Journal of Dentistry 1999; 27: 203-207.

21.Mertz-Fairhurst EG, Schuster GS, Fairhurst CW. Arresting caris by sealants: results of clinical study. Journal of the American Dental Association 1986; 112:194-196.

22.Paula Celiberti, and Adrian Lussi. Penetration ability and microleakage of fissure sealant applied on artificial and natural enamel fissure caries. Journal of Dentistry 2007; 35: 59-67.

23.Paula Celiberti, and Adrian Lussi. Use of a self-etching adhesive on previously etched intact enamel and its effect on sealant microleakage and tag formation. Journal of Dentistry 2005; 33; 163-171.

24.Ripa LW. Sealants revisited: an update of the effectiveness of pit and fissure sealants. Caries Research 1993; 27 (suppl.1):77-82.

25.Rix AM, Sams DR, Dickinson GL, Adair SM, Russell CM, Hoyle SL. Pit and fissure sealant application using a drying agent. American Journal of Dentistry 1994; 7(Abstr.3) :131-3.

26.Robert J and Feigal DDS. Sealants and preventive restorations: review of effectiveness and clinical changes for improvement. International Journal of Pediatric Dentistry1998; 20: 85-92.
27.Robert J. Feigal RG. Sealants and preventive restorations: review of effectiveness and clinical changes for improvement. Journal of American Academy of Pediatric Dentistry 1998; 20: 85-92.

28.ShuFen Chuanga, ChihHan Changa, Peter Yamanc, Li Tung Changd. Influence of enamel wetness on resin composite restorations using various dentine bonding agents: Part I effects on marginal quality and enamel microcrack formation. Journal of Dentistry 2006; 34: 343-351.

29.Solomon A, Beech DR. Bonding of composites to dentin using primers. Journal of Dental Materials 1985; 1: 79-82.

30.Taylor CL, Gwinett AG. A study of the penetration of sealants into pits and fissures. Journal of the American Dental Association 1973; 87:1188-1191.

31.Theodoridou-Pahini S, Tolidis K, Papadogiannis Y.Degree of microleakage of some pit and fissure sealants: an in vitro study. International Journal of Pediatric Dentistry 1996; 6: 173-176.

32. Youssef MN, Youssef FA, Souza-Zaroni WC, Turbino ML,Vieira MMF. Effect of enamel preparation method on in vitro marginal microleakage of a flowable composite used as pit and fissure sealant. International Journal of Pediatric Dentistry2006; 16:342-347. 